\title{
Catechol-0-Methyl Transferase (COMT) Enzyme Gene Variations in Patients with Idiopathic Scoliosis: A Clinical Chart Review
}

\author{
Mark W. Morningstar ${ }^{1}$, Megan N. Strauchman ${ }^{1}$, Clayton J. Stitzel2 ${ }^{2}$ Brian Dovorany ${ }^{3}$, Aatif Siddiqui ${ }^{4}$ \\ ${ }^{1}$ Natural Wellness \& Pain Relief Center, Grand Blanc, MI, USA \\ ${ }^{2}$ Lancaster Spinal Health Center, Lititz, PA, USA \\ ${ }^{3}$ ScoliSMART Clinics-Wisconsin, Green Bay, WI, USA \\ ${ }^{4}$ Esprit Wellness, New York, NY, USA \\ Email: *drmorningstar@nwprc.com
}

How to cite this paper: Morningstar, M.W., Strauchman, M.N., Stitzel, C.J., Dovorany, B. and Siddiqui, A. (2018) Catechol-O-Methyl Transferase (COMT) Enzyme Gene Variations in Patients with Idiopathic Scoliosis: A Clinical Chart Review. Open Journal of Genetics, 8, 35-41.

https://doi.org/10.4236/ojgen.2018.83004

Received: June 22, 2018

Accepted: July 10, 2018

Published: July 13, 2018

Copyright (c) 2018 by authors and Scientific Research Publishing Inc. This work is licensed under the Creative Commons Attribution International License (CC BY 4.0).

http://creativecommons.org/licenses/by/4.0/

\begin{abstract}
The effects of genomic variations and their associations with idiopathic scoliosis have been the discussion of many research trials. Previous investigations into catechol-o-methyl transferase (COMT) have identified its importance in dopamine and estrogen metabolism. While many genetic influences on idiopathic scoliosis have been observed, there has been no report of any relationship between COMT mutations and idiopathic scoliosis. The present study compared two groups of patients who received COMT genetic testing. The first group had a history of idiopathic scoliosis, while the other served as a non-scoliotic control group. The scoliosis group showed a positive COMT mutation in 33 out of 58 patients, while the control group showed 22/58 $(\mathrm{P}<$ $0.05)$. The homozygous genotype Met/Met occurred significantly more frequently in the scoliosis group. The increased incidence of COMT defects in the current scoliosis patient group warrants further study into how COMT variations may relate to the development or progression of idiopathic scoliosis.
\end{abstract}

\section{Keywords}

Enzyme, Genetics, Genomics, Neurotransmitters, Scoliosis

\section{Introduction}

There has been significant interest and investigation into the genetic contributions to the development and/or progression of idiopathic scoliosis. For example, genome-wide association studies [1] [2] and systematic reviews [3] [4] [5] 
have been published, in hopes of identifying any associations with adolescent idiopathic scoliosis. Although many single nucleotide polymorphisms have been previously identified, several of these have been excluded upon further investigation [6]. With many neuro-hormonal aspects of idiopathic scoliosis now identified [7], it is likely that interest in possible genetic contributions to idiopathic scoliosis will continue into the forseeable future.

Previous studies have described hormonal and neurotransmitter imbalances in patients with idiopathic scoliosis. For example, studies have suggested a relationship between melatonin and estrogen communication with osteoblast cells in patients with adolescent idiopathic scoliosis [8] [9]. At the core of many of these hormonal pathways is the methylation cycle. Previously, Morningstar et al. showed that patients with idiopathic scoliosis had a higher rate of methylenetetrahydrofolate reductase (MTHFR) polymorphism of the A1298C allele compared with non-scoliotic controls [10]. Another enzyme in the methylation cycle, catechol-O-methyl transferase (COMT) degrades catecholamine neurotransmitters (dopamine, norepinephrine, and epinephrine) into their constituent metabolites. This catecholamine degradation occurs by transferring a methyl group from a methyl donor, such as s-adenosyl methionine (SAMe) [Juarez]. COMT also breaks down estrone and estradiol into their respective metabolitesvia this same methyl donation and transfer process. Reduced estrogen breakdown activity by COMT is thought to contribute to cancer risk [11]. Given its importance in dopamine and estrogen metabolism, combined with previously published studies examining these chemicals relative to scoliosis etiopathogenesis [12] [13], this study examined the rate of occurrence of COMT polymorphisms in a cohort of patients with idiopathic scoliosis compared to a group of non-scoliotics. To date, no study has reported the presence of a COMT genetic variant in patients with idiopathic scoliosis.

\section{Materials and Methods}

Patient charts from a single medical office were examined and pulled for patients who presented with a history of scoliosis. Once these patient files were identified, those that fulfilled the following inclusion criteria were included in the present study: 1) Patients were diagnosed by the customary radiographic Cobb angle of $>10^{\circ}$, thus meeting the definition of scoliosis; 2) Patients had performed a genetic test from an online commercial genetics laboratory; 3) The patient's results had been interpreted by the same software program that analyzed the presence of COMT variants, and 4) all patients in both groups presented for initial treatment in the calendar years 2016 and 2017. Patient files were excluded if there was a history of a concurrent disorder that would characterize the scoliosis as neuromuscular or syndromic, such as cerebral palsy, Marfan's syndrome, Ehlers-Danlos syndrome, etc. For purposes of the present study, any treatment history, or treatment performed in the medical office from which the files were selected, was not considered. Based upon the above criteria, 58 total patient files 
were ultimately selected for study. All of these files were selected from the author's (MNS) integrative medicine clinic.

After these patient charts were selected, another group of 58 patient charts was identified. These patients had presented to the same medical office, had the same type of genetic testing performed for non-scoliosis diagnoses, but were negative for scoliosis. This group of charts served as the control group. For purposes of this study, patients were considered positive for the COMT genetic variant if they met the following positive criteria: 1 ) a double mutation of COMT Val158Met (homozygous positive), or 2) a single mutation (heterozygous positive). These results were recorded for all patients in both groups, and then compared using single factor ANOVA testing. Patients in both groups whose charts were selected subsequently provided written permission to use their non-identifying lab results and demographics.

\section{Results}

In the scoliosis group, 41 of the 58 patients were Caucasian, 7 were African-American, 5 were Hispanic, and 5 were Indian. The control group had an ethnic breakdown of 47 Caucasians, 3 African-Americans, 3 Indian-Americans, and 4 Hispanic-Americans. There were 46 females and 12 males in the scoliosis group; with 42 females and 16 males in the control group. The average age of the scoliosis group was 14 years, 4 months, while the average age of the control group was 29 years, 7 months. Table 1 provides a breakdown of this demographic data.

The scoliosis group was heterozygous or homozygous positive for a COMT variant in 33 out of 58 patients (57\%). Of these 33 patients, 14 were heterozygous (Val/Met expression) and 19 were homozygous (Met/Met). The non-scoliosis group was positive in 22 of 58 cases (38\%), with 15 being heterozygous and 7 homozygous. This information is shown in Table 2. These differences were statistically significant $(P<0.05)$. When comparing the differences of each genotype between the two groups using independent t-tests, the Val/Val (normal) genotype was significantly higher $(\mathrm{P}<0.05)$ than the scoliosis group. Conversely, the scoliosis group showed a significantly higher level $(\mathrm{P}<0.05)$ occurrence of the Met/Met genotype, 2.7 times more frequently than the control group. Both groups had a near equal occurrence of the Val/Met genotype (14 for scoliosis group, 15 for control group).

Specific to ethnicity, 3 of 5 Hispanics were COMT positive, along with 4 of 7 African-Americans, and 2 of 5 Indians. Figure 1 provides a breakdown of the number of COMT variants according to ethnicity. In the control group, $16 \mathrm{Cau}-$ casians, 3 African-Americans, 2 Indians, and 1 Hispanic patient were COMT positive.

The number of COMT variants was also categorized by gender. In the scoliosis group, 39 of the 41 COMT-positive patients were female; 2 were male. All 19 homozygous positive patients in the scoliosis group were female. The control 


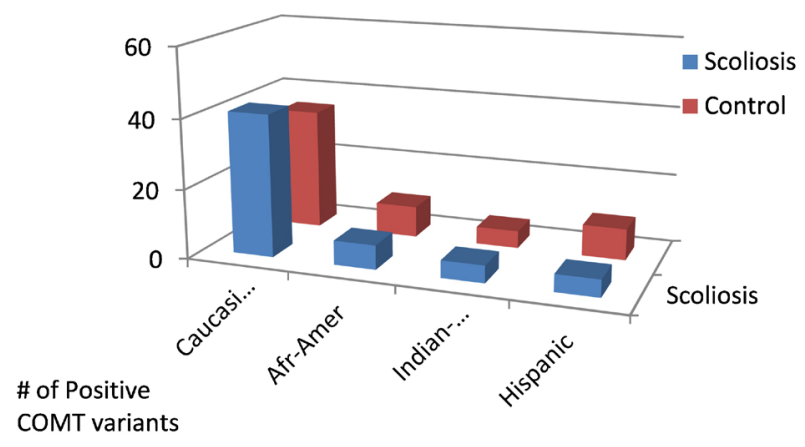

Figure 1. COMT variants by ethnicity.

Table 1. Demographic data for both groups.

\begin{tabular}{ccc}
\hline \multirow{2}{*}{ Demographics } & \multicolumn{2}{c}{ Group } \\
\cline { 2 - 3 } & Scoliosis & Control \\
\hline Age (years, months $)$ & 14,4 & 29,7 \\
Female & 46 & 42 \\
Male & 12 & 16 \\
Caucasian-American & 41 & 47 \\
African-American & 7 & 3 \\
Indian-American & 5 & 3 \\
Hispanic-American & 5 & 4
\end{tabular}

Table 2. Allele distribution of COMT Val158Met.

\begin{tabular}{cccc}
\hline \multirow{2}{*}{ Genotype } & \multicolumn{3}{c}{ Frequency (\%) } \\
\cline { 2 - 4 } & Val/Val & Val/Met & Met/Met \\
\hline Scoliosis Group & $25,(43 \%)$ & $14,(24 \%)$ & $19,(33 \%)^{\mathrm{a}}$ \\
Control Group & $36,(62 \%)^{\mathrm{a}}$ & $15,(26 \%)$ & $7,(12 \%)$ \\
\hline
\end{tabular}

a Statistically significant at $P<0.05$.

group had 16 COMT-positive patients, of which 11 were female an 5 male. Out of these 16 COMT-positive patients, 13 were Val/Met genotype and 3 were Met/Met.

A power analysis, given the mean and standard deviation, showed that a sample size of 56 would achieve $80 \%$ power.

\section{Discussion}

Morningstar et al. previously observed that urinary norepinephrine levels tend to be elevated in patients with idiopathic scoliosis compared to non-scoliotics [14]. Since it has been recognized that the Val allele expression may predispose patients to poorer motor learning [15], it is possible that this genetic variant may have an impact on the motor coordination in spinal muscle groups. Those with the COMT Met expression have reduced COMT activity, resulting in increased 
prefrontal levels of dopamine [15], which is directly converted into norepinephrine downstream. Therefore, this genetic variant may be responsible for the downstream elevation seen in peripheral urinary norepinephrine levels previously observed [14].

It may be noteworthy to discuss the potential implications of the distinct difference in the amount of homozygous variants in the scoliosis group $(n=19)$ compared to the control group $(n=7)$. Since idiopathic scoliosis appears to be multi-factorial [7], the Met/Met genotype needs to be further explored, as some of the etiological theories may be explained, at least in part, by this COMT genotype. If COMT variation is somehow connected to scoliosis causation or progression, then it is possible that the near equivalence of the heterozygous variant, between both groups in the present study, may have to occur in the presence of several other factors, such as other genetic variants or metabolic factors. There are multiple implications of the present study concerning the etiology and/or progression of idiopathic scoliosis. The COMT genetic variant has downstream effects on motor learning, norepinephrine metabolism, and estrogen metabolism. Since all of these have been associated with idiopathic scoliosis, future studies may look at how various lifestyle or functional medicine interventions can impact the onset or progression of idiopathic scoliosis by minimizing the impact of this genetic variant on downstream physiological pathways. Examples may include the introduction of methyl donors as supplements, promoting of diets that facilitate normal estrogen metabolism and excretion, and promotion of long-term motor memory activities that promote serotonin production to balance these inhibitory neurotransmitters with excitatory neurotransmitters (i.e. norepinephrine).

\section{Limitations}

Although the patients in the control group for the present study did not have scoliosis, they were nonetheless patients begin treated for other health issues and symptoms. Therefore, the control group used in this study may not necessarily be considered healthy controls. Additionally, it is possible that some of the symptoms for which they were being treated may have at least partially been the downstream result of the COMT variant in those control patients who were positive for it. Finally, while the scoliosis population selected in this study showed a significantly higher proportion of people with a positive COMT variant, this does not necessarily indicate that the COMT gene variant is a cause or contributing factor for the onset or progression of scoliosis. Selection bias may also affect the study results, although attempts were made to select every possible patient within the same time period.

\section{Conclusion}

In two different clinical samples of patient records, $57 \%$ of patients with a history of idiopathic scoliosis tested positive for a COMT Val158Met genetic variation, 
as compared to $38 \%$ of a non-scoliotic control group. Within these percentages, the homozygous Met/Met genotype variant occurred significantly more frequently in the scoliosis group as compared to the non-scoliotic group. Given the importance of the COMT enzyme on catecholamine metabolism, this study further identifies the relationship between abnormal neurotransmitter metabolism and idiopathic scoliosis. However, it is unknown how abnormal catecholamine metabolism relates to idiopathic scoliosis in terms of causation or effect. Further investigation may help to better identify any causal relationships.

\section{References}

[1] Zhu, Z., Xu, L., Leung-Sang Tang N, Qin, X., Feng, Z., Sun, W., et al. (2017) Genome-Wide Association Study Identifies Novel Susceptible Loci and Highlights Wnt/Beta-Catenin Pathway in the Development of Adolescent Idiopathic Scoliosis. Human Molecular Genetics, 26, 1577-1583. https://doi.org/10.1093/hmg/ddx045

[2] Ogura, Y., Kou, I., Japan Scoliosis Clinical Research Group, Scoliosis, J., Matsumoto, M., Watanabe, K., et al. (2016) Genome-Wide Association Study for Adolescent Idiopathic Scoliosis. Clinical Calcium, 26, 553-560.

[3] Yang, P., Liu, H., Lin, J. and Yang, H. (2015) The Association of rs4753426 Polymorphism in the Melatonin Receptor 1B (MTNR1B) Gene and Susceptibility to Adolescent Idiopathic Scoliosis: A Systematic Review and Meta-Analysis. Pain Physician, 18, 419-431.

[4] Grauers, A., Einarsdottir, E. and Gerdhem, P. (2016) Genetics and Pathogenesis of Idiopathic Scoliosis. Scoliosis and Spinal Disorders, 11, 45.

https://doi.org/10.1186/s13013-016-0105-8

[5] Wajchenberg, M., Astur, N., Kanas, M. and Martins, D.E. (2016) Adolescent Idiopathic Scoliosis: Current Concepts on Neurological and Muscular Etiologies. Scoliosis and Spinal Disorders, 11, 4. https://doi.org/10.1186/s13013-016-0066-y

[6] Janusz, P., Kotwicki, T., Małgorzata, K. and Szulc, A. (2012) Genetic Aspects of Idiopathic Scoliosis-Literature Review. Scoliosis, 7, O71.

https://doi.org/10.1186/1748-7161-7-S1-O71

[7] Burwell, R.G., Aujla, R.K., Grevitt, M.P., Dangerfield, P.H., Moulton, A. and Randell, T.L. (2009) Pathogenesis of Adolescent Idiopathic Scoliosis in Girls-A Double Neuro-Osseous Theory Involving Disharmony between Two Nervous Systems, Somatic and Autonomic Expressed in the Spine and Trunk: Possible Dependency on Sympathetic Nervous System and Hormones with Implications for Medical Therapy. Scoliosis, 4, 24. https://doi.org/10.1186/1748-7161-4-24

[8] Man, G.C., Wong, J.H., Wang, W.W., Sun, G.Q., Yeung, B.H. and Ng, T.B. (2011) Abnormal Melatonin Receptor 1B Expression in Osteoblasts from Girls with Adolescent Idiopathic Scoliosis. Journal of Pineal Research, 50, 395-402. https://doi.org/10.1111/j.1600-079X.2011.00857.x

[9] Letellier, K., Azeddine, B., Parent, S., Labelle, H., Rompré, P.H. and Moreau, A. (2008) Estrogen Cross-Talk with the Melatonin Signaling Pathway in Human Osteoblasts Derived from Adolescent Idiopathic Scoliosis Patients. Journal of Pineal Research, 45, 383-393. https://doi.org/10.1111/j.1600-079X.2008.00603.x

[10] Morningstar, M.W., Strauchman, M.N., Stitzel, C.J., Dovorany, B. and Siddiqui, A. (2017) Methylenetetrahydrofolate Reductase (MTHFR) Gene Mutations in Patients with Idiopathic Scoliosis: A Clinical Chart Review. Open Journal of Genetics, 7, 62-67. https://doi.org/10.4236/ojgen.2017.71006 
[11] Juarez, B. and Han, M.H. (2016) Diversity of Dopaminergic Neural Circuits in Response to Drug Exposure. Neuropsychopharmacology, 41, 2424-2446. https://doi.org/10.1038/npp.2016.32

[12] Dalessandri, K.M., Firestone, G.L., Fitch, M.D., Bradlow, H.L. and Bjeldanes, L.F. (2004) Pilot Study: Effect of 3,3'-Diindolylmethane Supplements on Urinary Hormone Metabolites in Postmenopausal Women with a History of Early-Stage Breast Cancer. Nutrition and Cancer, 50, 161-167.

https://doi.org/10.1207/s15327914nc5002_5

[13] Morningstar, M. (2016) Neurotransmitter Status and Idiopathic Scoliosis: A Commentary on Pathways, Testing, Clinical Utility, and Treatment. Current Pediatric Research, 20, 14-19.

[14] Morningstar, M.W., Siddiqui, A., Dovorany, B. and Stitzel, C.J. (2014) Can Neurotransmitter Status Affect the Results of Exercise-Based Scoliosis Treatment? Results of a Controlled Comparative Chart Review. Alternative \& Integrative Medicine, 3 , 177.

[15] Morningstar, M. (2013) Neurotransmitter Patterns in Patients with Adolescent Idiopathic Scoliosis (AIS). Scoliosis, 8, O1. https://doi.org/10.1186/1748-7161-8-S2-O1

[16] Noohi, F., Boyden, N.B., Kwak, Y., Humfleet, J., Burke, D.T., Müller, M.L.T.M., et al. (2014) Association of COMT val158met and DRD2 G>T Genetic Polymorphisms with Individual Differences in Motor Learning and Performance in Female Young Adults. Journal of Neurophysiology, 111, 628-640. https://doi.org/10.1152/jn.00457.2013 